\title{
DEMONSTRATIO MATHEMATICA
}

Vol. XII

No 1

1979

'Zygmunt Pachulski

\section{ON THE RADIUS OF CONVEXITY FOR CERTAIN FAMILY OF FUNCTIONS MEROMORPHIC IN UNIT DISC}

1. Let $q$ denote the family of functions of the form

$$
p(z)=1+p_{1} z+p_{2} z^{2}+\cdots
$$

holomorphic in the disc $K=\{z:|z|<1\}$ and for eviery $z \in K$ satisfying the condition re $p(z)>0$.

Let the symbol $P(m)$ denote the subclass of m-symetric functions of the family , where $m \geqslant i$ is a fixed natural number.

Denote by $\Sigma$ the family of functions $F$ meromorphic and univalent in the disc $K$ and which in the neighbourhood $z=0$ have the expansion of the form

$$
w=F(z)=\frac{1}{z}+a_{0}+a_{1} z+a_{2} z^{2}+\cdots
$$

In the farily $\Sigma$ we distinguish two following subclarses; subclass $\Sigma^{*}$ of the fur.stions $w=F(z)$ mapping the disc $\mathrm{K}$ snto reg:ons whose $c$ omplements to the plane are starlike reoion in alation to the foint $w=0$, and subcrass $\Sigma_{\alpha}^{*}$ of the functions $w=F(z)$, such that, for an arbitraxy fixed $\alpha, 0 \leqslant \alpha<1$, and for every $z \leqslant Z$, the condition is satisfied (i. i)

$$
\text { re }\left[-2 F^{\prime}(z) / F(z)\right]>\alpha \text {. }
$$

It fcllows that $F \in \Sigma_{\alpha}^{*}$ if and orly if tilere is a function $p \in \Psi$ such that for every $z \in K$, the condi: $n$ : 


$$
-z F^{\prime}(z) / F(z)=(1-\alpha) p(z)+\alpha \quad(\operatorname{see}[4], p .518)
$$

Is satisfied.

Finaliy let $\Sigma_{\alpha}^{*}(\beta)$ denote the class of functions $F \in \Sigma$ for which the condition

$$
-\frac{z F^{\prime}(z)}{F(z)}=[(1-\alpha) p(z)+\alpha]^{\beta}, z \in \mathbb{B}
$$

Is satisfied, where $p \in q$ and $0 \leqslant \alpha<1,0<\beta \leqslant 1$ are arotrary eixed real numbers.

\section{Denote}

$$
r(F)=\sup \left\{r: r e\left[-\left(1+\frac{z F^{\prime \prime}(z)}{r^{(}(z)}\right)\right]>0,|z|<r\right\} \text {. }
$$

The number $r^{C}\left[\Sigma_{\alpha}^{*}(\beta)\right]=\inf \left\{r(F): \mathbb{F} \in \Sigma_{\alpha}^{*}(\beta)\right\}$ will be called the radius of convexity of the family $\Sigma_{\alpha}^{*}(\beta)$. It is easy to show that the family $\Sigma_{\alpha}^{*}(\beta)$ is compact, hence the radius of convexity of the fanily $\Sigma_{\alpha}^{*}(\beta)$ is equal to the greatest value of $r, 0<r \leqslant 1$ for which

$$
r e\left[-\left(1+\frac{z F^{\prime \prime}(z)}{F^{\prime}(z)}\right)\right] \geqslant 0
$$

for every $F \in \Sigma_{\alpha}^{*}(\beta)$ and $z$, where $|z| \leqslant r$.

Thus the radius of convexity $r^{c}\left[\Sigma_{\alpha}^{*}(\beta)\right]$ is equal to the smaliest root $r_{0}, 0<r_{0} \leqslant 1$, of the equation $\omega(x)=0$, where

$$
\omega(r)=\min \left\{r e\left[-\left(1+\frac{z F^{\prime \prime}(z)}{H^{*}(z)}\right)\right]:|z|=r<1, F \in \Sigma_{\alpha}^{*}(\beta)\right\} .
$$

From condition (1.2) it follows that for every $F \in \Sigma_{\alpha}^{*}(\beta)$ there exists a function $p \in \mathscr{F}$ such that, for every $z \in K$, the equality is satisfied

$$
-\left[1+\frac{z F^{\prime \prime}(z)}{F^{\prime}(z)}\right]=[(1-\alpha) p(z)+\alpha]^{\beta}-\frac{\beta(1-\alpha) z p^{\prime}(z)}{(1-\alpha) p(z)+\alpha} .
$$


From the last two equalities (see[3]) we obta:

$$
\text { (2.2) } \begin{aligned}
\omega(r) & =\min \left\{r e \left[\left((1-\alpha) p^{*}(z)+\alpha\right)^{\beta}-\frac{\beta(1-\alpha) z p^{*}(z}{(1-\alpha) p^{*}(z)+\alpha}:|z|=\right.\right. \\
& \left.=x<1, p^{*} \in P_{2}\right\},
\end{aligned}
$$

where $P_{2}$ is the family of functions of the form

$$
p^{*}(z)=\frac{1+\lambda}{2} \frac{1+\varepsilon_{1} z}{1-\varepsilon_{1} z}+\frac{1-\lambda}{2} \frac{1+\varepsilon_{2}{ }^{z}}{1-\varepsilon_{2}{ }^{2}},
$$

$-1 \leqslant \lambda \leqslant 1,\left|\varepsilon_{1}\right|=\left|\varepsilon_{2}\right|=1$.

Making use of Lomma 1 and 2 (see [1], p.70) and assuming $h=\frac{\alpha}{1-\alpha}$ from $(2.2)$ we obtain

$$
\text { (2.3) } \begin{aligned}
\omega(r) & =\min \left\{\operatorname { r e } \left[\left(\frac{p^{*}(z)+h}{1+h}\right)^{\beta}-\beta \frac{\left(p^{*}(z)\right)^{2}-1}{2\left(p^{*}(z)+h\right)}+\right.\right. \\
& \left.\left.+\beta \frac{\left(\rho^{2}-\left|p^{*}(z)-c\right|^{2}\right) \eta}{2\left(p^{*}(z)+h\right)}\right],|z|=r<1, \quad p^{*} \in q_{2}\right\},
\end{aligned}
$$

where $z=r e^{1 \varphi}, \quad 0 \leqslant r<1, \quad 0 \leqslant \varphi \leqslant 2 \pi$,

$$
c=\frac{1+r^{2}}{1-r}, \quad \rho=\frac{2 r}{1-r^{2}}
$$

$\eta=\eta_{1} \eta_{2}$ at the same time

$$
\eta_{k}=\varepsilon_{k} e^{1 \varphi} \frac{1-\bar{\varepsilon}_{k} e^{-1 \varphi_{r}}}{1-\varepsilon_{k} e^{1 \varphi} r} ; k=1,2 ;\left|\varepsilon_{1}\right|=\left|\varepsilon_{2}\right|=1
$$

Assuming $\mathrm{p}^{*}\left(r \mathrm{e}^{1 \varphi}\right)+\mathrm{h}=\mathrm{se}^{1 t}$, im $\mathrm{t}=0$ and taking into acco unt the inequality re $\left(e^{-i t} \eta\right) \geq-1$ and the equality

$$
\rho^{2}-\left|s e^{1 t}-n-c\right|^{2}=2 n s \cos t+2 \cos \cos t-s^{2}-h^{2}-2 h c-1
$$

from $(2.3)$, we obtain $\omega(r) \geqslant \underset{\rho, t}{\min } L(s, t)$ where the function 
(2.5) $L(\theta, t)=\frac{\theta^{\beta} \cos \beta t}{(1+h)^{\beta}}+\beta \frac{1-h^{2}-s^{2}}{2 s} \cos t+\beta h-\beta(h+c) 00 a t+$ $+\beta \frac{s^{2}+h^{2}+2 h c+1}{2 s}$

is defined in the disc $D_{r}$, where

$$
\begin{aligned}
D_{r}=\{ & (s, t): s^{2}-2 s(c+h) c o s t+h^{2}+2 h c+1 \leqslant 0 \\
& \left.-\frac{\pi}{2} \leqslant t \leqslant \frac{\pi}{2}, c+h-\rho \leqslant s \leqslant c+h+\rho\right\} .
\end{aligned}
$$

Direct calculations (see[4], p.524) show that the function $I(s, t)$ can reach the minimum only at those points $(s, t) \in D_{r}$ for which $t=0$. The problem of determining the minimum of the function $\omega(r)$ is thus reduced to determining the minimum of the function $L_{1}(s)=L(s, 0)$ in the interval $s \in[c+h-\rho$, $c-h+Q]$.

We have the following lemmas.

$\mathrm{L} e \mathrm{~m}$ in a 1 . The function

$$
I_{\eta}(s)=\frac{s^{\beta}}{(1+h)^{\beta}}+\beta \frac{1+h c}{s}-\beta c
$$

reaches the absolute minimum on the interval $[c+h-\rho$, $c+h+\rho]$ in the point

$$
s_{1}=c+h+\rho
$$

if the inequality $s_{1} \leqslant s_{2}$ is satisfied, where

$$
s_{2}=(1+h)^{\frac{\beta}{1+\beta}}(1+h c)^{\frac{1}{1+\beta}} .
$$

If, however, $s_{1}>s_{2}$, then the function $I_{1}(s)$ reaches this minimum in the point $s_{2}$. The minimum respectiveig mounts to 
(2.8) $K_{1}(r)=N_{1}(r ; \beta, h)=\frac{(c+h+\rho)^{1+\beta}+\beta(1+h)^{\beta}(1+h c)}{(1+h)^{\beta}(c+h \cdot+\rho)}-\beta c$

$$
H_{2}(r)=M_{2}(r ; \beta, h)=(1+\beta)\left(\frac{1+h c}{1+h}\right)^{\frac{\beta}{1+\beta}}-\beta c \text {. }
$$

$I \in m$ m a 2. For every function $F \in \Sigma_{\alpha}^{*}(\beta)$ and for every fixed $z,|z|=r, 0<r<1$, and at fixed $\alpha$ and $\beta$ the acute estimate holàs:

(2.10)

$$
r e\left\{-\left[1+\frac{z F^{\prime \prime}(z)}{F^{\prime}(z)}\right]\right\} \geqslant\left\{\begin{array}{ll}
M_{1}(x) & \text { for } s_{1} \leqslant s_{2} \\
M_{2}(x) & \text { for } s_{1}>s_{2}
\end{array},\right.
$$

where $s_{1}, s_{2}, M_{1}(r), M_{2}(r)$ are respectively defined by the formulas (2.6) - (2.9). The equalities jn the estimations (2.10) are realised respectively by functions of the form

(2.11) $F_{1}(z)=\frac{1}{z} \exp \int_{0}^{z}\left[1-\left(\frac{1+(1-2 \alpha) e^{-i \varphi_{p}}}{1-e^{-1 \varphi} z}\right)^{\beta}\right] \frac{d z}{z}$

(2.12; $\quad F_{2}(z)=\frac{1}{2} \exp \int_{0}^{z}\left[1-\left(\frac{1+2 K(1-\alpha) e^{-i \varphi} z+(1-2 \alpha) e^{-2 i \varphi_{z}} z^{\beta}}{1-e^{-2 i \varphi_{z} 2}}\right)\right] \frac{d z}{z}$.

where $0 \leqslant \varphi \leqslant 2 \pi$, however

$$
K=\frac{\left(s_{2}-h\right)\left(1-r^{2}\right)-\left(1+r^{2}\right)}{2 r} .
$$

3. We have the following theorem.

I h e o r e m. Denote by $r_{0}$ the only root of the truati,n (3.1) $\left(\frac{1+\beta}{\beta}\right)^{\frac{1+\beta}{\beta}} \beta(1+r)\left(1-r^{2}\right)^{\frac{1}{\beta}}+\left(1+r^{2}:\left[r^{c}-(1+\beta) r-\beta\right]=0\right.$ in the interval $(0,1)$ and let $\alpha_{0}$ desate the expression 


$$
\alpha_{0}=\frac{\left(1+r_{0}\right)\left[r_{0}^{2}-(2 \beta+2) r_{0}+1\right]}{2 r_{0}\left[r_{0}^{2}-(1+\beta) r_{0}-\beta\right]},
$$

where $\beta \in(0 ; 1]$, is an arbitrary fixed real number. The radius of convexity of the family $\Sigma_{\alpha}^{*}(\beta)$ is given by the formula

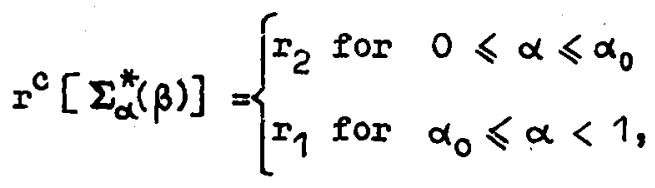

where $r_{2}$ is equal to the only root in the interval $(0,1)$ of the equation

$$
\text { (3.4) }\left(\frac{i+\beta}{\beta}\right)^{1+\beta}\left(1-r^{2}+h+h r^{2}\right)\left(1-r^{2}\right)-\left(1+r^{2}\right)^{1+\beta}(1+h)^{\beta}=0
$$

and $r_{1}$ is equal to the only root in the interval $(0,1)$ of the equation

$$
\text { (3.5) }(1+r+h-h r)^{1+\beta}(1-r)^{1-\beta}-2 \beta(1+h)^{\beta} r=0 \text {. }
$$

The forms of the extremal functions are respectively given by (2.12) and (2.11).

F $r \circ \circ$ f. From Lemma 2 and from the formula (2.1) it follows that the radius of convexity of the family $\Sigma_{\alpha}^{*}(\beta)$ is equal to the root of the equation $M_{1}(r)=0$ if $s_{1} \leqslant s_{2}$, and it is equal to the root of the equation $M_{2}(r)=0$ if $s_{1}>s_{2}$.

It can be proved that the value $\alpha_{0}$ given by formulas (3.1) and (3.2) satisfies the inequality $\frac{1}{2}<\alpha_{0}<1$ for every $\beta \in(0,1]$ and that for arbitrary fixed $\beta \in(0,1]$ and $\alpha \in\left[0, \alpha_{0}\right)$ the inequality $s_{1}>\theta_{2}$ holds $s_{3}$ for arbitrary fixed $\beta \in(0,1]$ and $\alpha \in\left(\alpha_{0}, 1\right)$ the inequality $s_{1}<s_{2}$ holds, however for $\alpha=\alpha_{0}$ we have the equality $s_{1}=s_{2}$. Solving the equations $M_{2}(r)=0$ and $M_{1}(r)=0$ respectively, we obtain (3.3). 
C o r o 1 I a $r$. Assuming $\beta=1$ we obtain, from the last theorem, the radius of convexity of the family $\Sigma_{\alpha}^{*}$ mariked by V.A. Zmorovic (see [4], p.524); assuming $\alpha=0$ we obtain the radius of convexity of the family $\Sigma{ }^{*}(\beta)$ for $m=1$, $r^{c}\left[\Sigma^{*}(\beta)\right]=\frac{1}{\sqrt{1+2 \beta}}($ see [1], p.81-82); however in the case when $\alpha=0$ and $\beta=1$ we obtain $M$. Robertson's result for the family $\Sigma^{*}, r=\frac{1}{\sqrt{3}}$ (see [3], p.239).

\section{REFERENCES}

[1] Z. P a c h u 1 s $k 1$ : On extremal problems of some famtly of meromorphic functions, Acta Univ.Lod., Seria II, Nr 17 (1977) 63-84.

[2] Ch. P o m m e r e n k e; On meromorphic starilke functions Pac. J. Math., 13 (1963) 221-235.

[3] M.S. R $\mathrm{O} b$ e $\mathrm{r} s$ o $n$; extremal problems for analytic functions with positive real part and applications, Trans. Amer.llath.Soc., 2(1963) 236-253.

[4] J.A. Z m $0 \mathrm{r} \circ \mathrm{v} i \check{c}$; 0 Eranicach wypukjosti zvezdnych funkcij porjadka $\alpha \mathrm{v}$ kruge $|z|<1$ i krugovoj oblasti $\therefore<|z|<1$, Mat.Sb., N.63(110), no 4 (1965) 518-526.

INSP ITUTE CF MATHEMATICS, PEDAGOGICAI, UNIVERSITY, CZESTOCHONA Received Miarch 31, 1978. 
